BULL. AUSTRAL. MATH. SOC.

\title{
A note on subnormality
}

\section{T.A. Peng}

Let $H$ be a subgroup of a finite group $G$ and let $S$ be a set of generators of $B$. We prove that if $G$ is soluble, then $H$ is subnormal in $G$ if and only if there exists an integer $n$ such that for each $g$ in $G$ and $a$ in $S$ the commutator $[g, \underbrace{a, \ldots, a}_{n}]$ lies in $H$. This criterion for subnormality is also valid for soluble groups satisfying the maximal or the minimal condition on subgroups.

Let $G$ be a group. For elements $x$ and $y$ of $G$ we write $[x, y]$ for the commutator $x^{-1} y^{-1} x y$ and define $\left[x, n^{y}\right]$ inductively by $\left[x, 0^{y}\right]=x$ and $[x, n+1]=\left[\left[x, n^{y}\right], y\right]$ for $n \geq 0$. Let $H$ be a subgroup of $G$ and let $S$ be a set of generators of $H$. If $H$ is subnormal in $G$, then there exists an integer $n \geq 0$ such that for each $g$ in $G$ and $a$ in $S$ the commutator $[g, n a]$ lies in $H$. For an arbitrary group $G$ the converse is false. (For an example, see [4], p. 230.) Is the converse true if $G$ is a finite group? This question was raised by Wielandt in a lecture at the Mathematics Institute, Warwick, in early 1973. (See also [6], p. 203.) In this note we give a partial answer to Wielandt's question by proving that the converse is true if $G$ is a finite soluble group. We shall deduce this and a number of other results of a similar kind from a more general theorem on subnormality in soluble groups which are not necessarily finite.

We use standard notation. Let $B$ be a subgroup of a group $G$ and let $S$ be a set of generators of $H$. We shall say that the set $S$

Received 16 March 1976. 
satisfies the condition $C_{n}$ (in $G$ ) if there exists an integer $n \geq 0$ such that for each $g$ in $G$ and $a$ in $S$ the commutator $[g, n a$ lies in $H$. We write $H$ sn $G$ to indicate that $H$ is a subnormal subgroup of $G$.

THEOREM. Let $G$ be a soluble group and let $H$ be a subgroup of $G$. Suppose that $H$ is generated by a finite normal subset $S$ (that is, $S^{h}=S$ for all $h$ in $H$ ). If $S$ satisfies the condition $c_{n}$ for some integer $n \geq 0$, then $H$ is subnormal in $G$.

Proof. We first prove the special case where $S$ consists of a single conjugacy class of $H$. We do this by induction on the derived length $d(G)$ of $G$.

The result is trivially true if $d(G)=1$ (that is, if $G$ is abelian). So we assume that $d(G) \geq 2$. We also assume that $n \geq 1$ since $n=0$ implies that $H=G$.

Let $N$ be the last non-trivial term of the derived series of $G$. Clearly $S N / N$ generates $H N / N$ and consists of a single conjugacy class. Since $S N / N$ satisfies the condition $C_{n}$ in $G / N$ and $d(G / N)=d(G)-1$, it follows by induction that $H N / N$ sn $G / N$ or $H N$ sn $G$. It is therefore sufficient to prove that $H$ sn $H N$.

Let $M=H \cap N$. Then $M \triangleleft H N$. Since $H$ sn $H N$ if and only if $H / M$ sn $H N / M$, there is no loss of generality if we assume that $M=1$. Under this assumption we have $H=H / M \cong H N / N$, so that the derived length $d(H)$ of $H$ is at most $d(G)-1$. (The derived length of $H N$ may well be $d(G)$, but this will not concern us.)

Let $g \in N$ and $a \in S$. Then $[g, i a] \in N$ for all $i \geq 0$. This together with the condition $C_{n}$ and the assumption $M=1$ yields $[g, n a]=1$. Let $m \leq n$ be the smallest positive integer such that $\left[g, m^{a}\right]=1$ for all $g \in N$ and $a \in S$. If $m=1$, then $[N, H]=1$, so that $H \triangleleft H N$. We may assume that $m>1$.

By the choice of $m$ there exist non-identity elements $g \in N$ and $a \in S$ such that $\left[g, m^{a}\right]=1$ but $[g, m-1] \neq 1$. Putting 
$x=\left[g, m_{-1} a\right]$, we get $[x, a]=1$. We show that $x$ centralizes the whole of $H$.

Let $K=B \cap H^{X} . \quad K$ is non-trivial since it contains the element a. Suppose that $K$ is a proper subgroup of $H$. Let $S_{\perp}=\left\{a^{h} \mid h \in K\right\}$ and let $H_{1}=\left(S_{1}\right)$. Since $S_{1} \leq H^{x}$, we have $S_{1}^{x^{-1}} \leq H$, so that $\left[x^{-1}, S_{1}\right] \leq H$. Since $x$ lies in the normal subgroup $N$, we have also $\left[x^{-1}, S_{1}\right] \leq N$. Therefore $\left[x^{-1}, S_{1}\right] \leq H \cap N=1$. Hence $x$ centralizes $S_{1}$

Let $S_{1}$ and $H_{1}$ be as above. If the subset $S_{i}$ and the subgroup $H_{i}$ have already been defined for $i \geq 1$, then let $S_{i+1}=\left\{a^{h} \mid h \in H_{i}\right\}$ and let $H_{i+1}=\left\langle S_{i+1}\right\rangle$. Since $S$ is finite, there exists an integer $r \geq 1$ such that $S_{r+1}=S_{r}$. But then $S_{r}=\left\{a^{h} \mid h \in H_{r}\right\}$. Hence $H_{r}$ is generated by a subset $S_{r}$ consisting of a single conjugacy class.

Let $h \in H$ and $b \in S_{r}$. Then it follows from the condition $C_{n}$ that $\left[h^{x^{-1}}, n^{b}\right] \in H$. Since $x$ commutes with $b$, we have

$$
\left[h, n^{b}\right]=\left[h^{x^{-1}}, n^{b}\right]^{x} \in H^{x}
$$

But $\left[h, n^{b]}\right.$ is an element of $H$. Hence $\left[h, n^{b}\right] \in H \cap H^{x}=K$. Since $H_{1} \triangleleft K$ and $H_{i+1} \triangleleft H_{i}$ for $i=1, \ldots, r-1$, we have

$$
\left[h,{ }_{n+r}^{b}\right]=\left[\left[h, n^{b]}, r^{b}\right] \in H_{r}\right. \text {. }
$$

Thus as a set of generators of $H_{p}$ the subset $S_{r}$ satisfies the condition $C_{n+r}$ in $H$. Since $d(H)$ is at most $d(G)-1$, it follows by induction that $H_{r}$ sn $H$. This implies that $S$ lies in a proper normal subgroup of $H$. This contradiction shows that $K=H$. But then $H \leq H^{x}$ and 
consequently $\left[x^{-1}, H\right] \leq H \cap N=1$, which proves that $x$ centralizes $H$. Let $N_{1}=C_{N}(H)$ be the centralizer of $B$ in $N \cdot N_{1}$ is non-trivial since it contains the element $x$. In fact, our argument shows that $N_{1}$ contains the element $\left[g, m_{-1} a\right]$ for each $g \in N$ and $a \in S$. Since $N$ is abelian, $N_{1}$ lies in the centre of $H N$ and is therefore normal in $H N$. For any $g \in N$ and $a \in S$, write $\bar{g}=g N_{1}$ and $\bar{a}=a N_{1}$. Then in the factor group $H N / N_{1}$ we have $\left[\bar{g}, m_{-1} \vec{a}\right]=1$ for all $\bar{g} \in N / N_{1}$ and $\bar{a} \in S N_{1} / N_{1}$. We may therefore repeat the above argument to obtain a subgroup $N_{2} / N_{1}$ of $N / N_{1}$ given by $N_{2} / N_{1}=C_{N / N_{1}}\left(H N_{1} / N_{1}\right)$. If $N_{2}$ is a proper subgroup of $N$, then we repeat the argument again with $N_{2}$ in place of $N_{1}$. Continuing in this way we obtain subgroups

$N_{0}=1, N_{1}, N_{2}, \cdots$ of $N$ with $N_{i+1} / N_{i}=C_{N / N_{i}}\left(H N_{i} / N_{i}\right)$ for

$i=0,1,2, \ldots$. Clearly the process must come to a stop after at most $m$ repetitions. So there exists an integer $I \leq s \leq m$ such that $N_{s}=N$. Since $N_{i+1} / N_{i}$ lies in the centre of $H N / N_{i}$ for $i=0,1,2, \ldots$, it follows that $H N_{i} / N_{i}$ is normal in $H N_{i+1} / N_{i}$ or $H N_{i} \triangleleft H N_{i+1}$. Hence $H$ sn $U N$. This completes the proof of the special case.

We now prove the general case where $S$ is a finite normal subset of $H$. We use induction on the order $|S|$ of $S$. Suppose that $S$ is a union of $t \geq 1$ conjugacy classes of $H, D_{1}, \ldots, D_{t}$ say. If $t=1$, we are back in the special case. So we assume that $t>1$. Let $E_{i}=\left\langle D_{i}\right\rangle$ for $i=1, \ldots, t$. Then $E_{i} \triangleleft H$, so that $D_{i}$ satisfies the condition $C_{n+1}$ for each $i$. Since $D_{i}$ is a finite normal subset of $E_{i}$ and $\left|D_{i}\right|<|S|$, it follows by induction that $E_{i}$ sn $G$ for each $i$. A result of Robinson ([5], Lemma 2.2) now gives $H=\left\langle E_{1}, \ldots, E_{t}\right\rangle$ sn $G$. This completes the proof of the theorem.

COROLLARY 1. Let $G$ be a soluble group and let $H$ be a finite subgroup of $G$. If $H$ is generated by a subset $X$ which satisfies the 
condition $C_{n}$ for some integer $n \geq 0$, then $H$ is subnormal in $G$.

Proof. Let $S=\left\{a^{h} \mid a \in X\right.$ and $\left.h \in H\right\}$. Then $S$ is a finite normal subset generating $H$ and satisfying the condition $C_{n}$. It follows from the theorem that $H$ is subnormal in $G$.

Let $G$ be a soluble group satisfying the maximal condition on subgroups and let $B$ be a subgroup of $G$. Then Kegel has shown in [3] that $H$ is subnormal in $G$ if and only if for each normal subgroup $N$ of $G$ with $G / N$ finite $H N / N$ is subnormal in $G / N$. This together with Corollary 1 gives the following result.

COROLLARY 2. Let $G$ be a soluble group satisfying the maximal condition on subgroups and let $H$ be a subgroup of $G$. If $H$ is generated by a subset $S$ which satisfies the condition $C_{n}$ for some integer $n \geq 0$, then $H$ is subnormal in $G$.

There is a corresponding result for soluble groups satisfying the minimal condition on subgroups. But we can prove more. Since it is well known that a soluble group satisfying the minimal condition on subgroups is abelian-by-finite, the corresponding result is contained in the following corollary.

COROLLARY 3. Let $G$ be a soluble abelian-by-finite group and let $H$ be a subgroup of $G$. If $H$ is generated by a subset $S$ which satisfies the condition $C_{n}$ for some integer $n \geq 0$, then $H$ is subnormal in $G$.

Proof. Let $N$ be an abelian normal subgroup of $G$ such that $G / N$ is finite. Then $H N / N$ is finite. By Corollary $1, H N / N$ sn $G / N$ or $H N$ sn $G$. Since $N$ is abelian, $H \cap N$ is normal in $H N$. Thus $H / H \cap N$, which is isomorphic to $H N / N$, is a finite subgroup of $H N / H \cap N$. By Corollary 1 again, $H / H \cap N \operatorname{sn} H N / H \cap N$ or $H \operatorname{sn} H N$. Hence $H \operatorname{sn} G$.

Another easy consequence of our theorem is the following result of Gruenberg ([2], Lemma 12): if $a$ is a bounded left Engel element of a soluble group $G$ that is, there exists an integer $n \geq 0$ such that $[g, n a]=1$ for all $g \in G$ ), then $a$ sn $G$. Using this result and a well-known theorem of Baer ([1], $\$ 3$, Satz 3) on the join of cyclic subnor subnormal subgroups and an argument similar to that of Corollary 3 above, 
we can prove easily the following result.

COROLLARY 4. Let $G$ be an extension of an abelian group by a finitely generated nilpotent group and let $H$ be a subgroup of $G$. If $H$ is generated by a subset $S$ which satisfies the condition $C_{n}$ for some integer $n \geq 0$, then $H$ is subnormal in $G$.

It may be worth remarking that the words "finitely generated" in Corollary 4 cannot be omitted. In fact, for each prime $p$ there exists a metabelian p-group with an abelian subgroup $H$ such that $H$ itself satisfies the condition $C_{p}$ but is not subnormal in $G$ ([4], p. 230).

\section{References}

[1] Reinhold Baer, "Nilgruppen", Math. Z. 62 (1955), 402-437.

[2] K.W. Gruenberg, "The Engel elements of a soluble group", IlZinois $J$. Math. 3 (1959), 151-168.

[3] Otto H. Kegel, "Über den Normalisator von subnormalen und erreichbaren Untergruppen", Math. Ann. 163 (1966), 248-258.

[4] T.A. Peng, "A criterion for subnormality", Arch. Math. (Basel) 26 (1975), 225-230.

[5] Derek S. Robinson, "Joins of subnormal subgroups", IZZinois J. Math. 9 (1965), 144-168.

[6] Helmut Wielandt, "Kriterien fur Subnormalitat in endlichen Gruppen", Math. 2. 138 (1974), 199-203.

Department of Mathematics, University of Singapore, Singapore. 